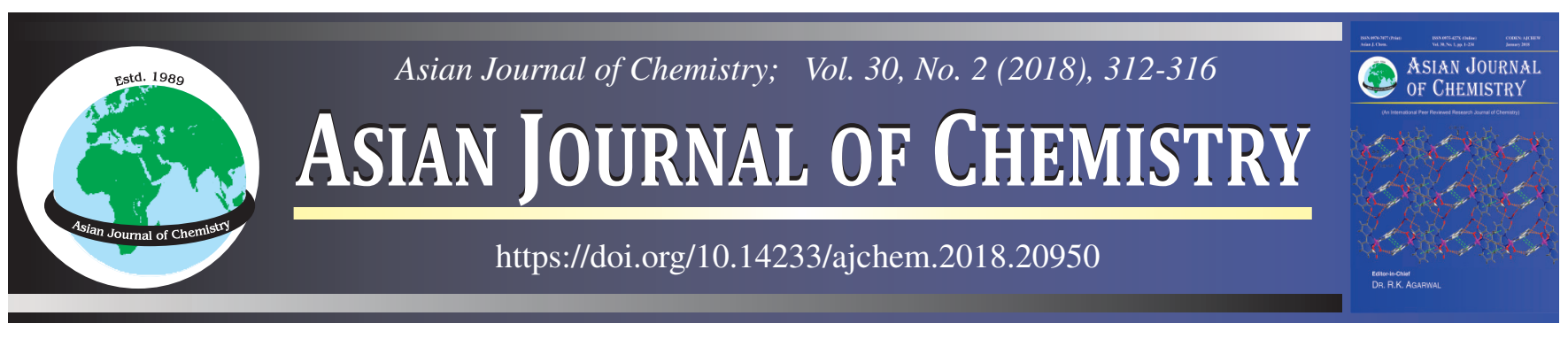

\title{
Synthesis, Characterization and Antiinflammatory Activity of Chalcone Derivatives Linked with Apocynin and 5-Nitrofuran Moiety
}

\author{
A.L.V. Kumar Reddy ${ }^{1, *}$ and NiRen E. Kathale ${ }^{2}$
}

${ }^{1}$ Department of Chemistry, Gondwana University, Gadchiroli-442 605, India

${ }^{2}$ Department of Chemistry, Sardar Patel Mahavidyalaya, Chandrapur-442 402, India

*Corresponding author: E-mail: alvkumar2016@gmail.com

The present paper describes the synthesis of some new chalcone derivatives i.e. 1-[3-methoxy-4-(5-nitro-furan-2-ylmethoxy)-phenyl]-3(substituted phenyl)-propenone derivatives (9A-9K) from furfural and apocynin as starting materials. Claisen-Schmidt reaction of 1-(4((5-nitrofuran-2-yl)methoxy)-3-methoxyphenyl)ethanone (7) with aromatic aldehydes (8A-K) under solvent free conditions using solid $\mathrm{NaOH}$ as catalyst at room temperature resulted in the formation of chalcone derivatives $(\mathbf{9 A - 9 K})$ in $86-96 \%$ yield. These compounds were characterized by ${ }^{1} \mathrm{H}$ NMR, Mass and IR spectroscopy and were evaluated for their anti-inflammatory activity.

Keywords: Anti-inflammatory activity, Apocynin, Chalcones, Furfural, Synthesis.

ᄂ - - - - - - - - - - - - - - - - - - - - - - - - - - - -

\section{INTRODUCTION}

Chalcone or (E)-1,3-diphenyl-2-propene-1-one, is an imperative chemotype that has fascinated enormous research interest for decades due to the elevated natural abundance of chalcone compounds, their easy synthesis and most prominently their varied biological activities. A number of chalcone compounds have been marketed or clinically tested for a variety of health conditions e.g., (i) diuretic-metochalcone/choleretic, (ii) antiulcersofalcone/mucoprotective (iii) hesperidin methyl-chalconevascular protective [1]. They are significant as structural motifs among biologically active molecules and also for combinatorial assembly of heterocyclic scaffolds [2-4]. Chalcones containing several functional groups showed a wide spectrum of biological activities such as antileishmanial [5,6], antimalarial [5,7], anticancer [8,9], anti-HIV [10], antioxidant [11], inflammatory [12] antiprotozoal [13], antiulcer [14] and antimicrobial $[15,16]$ activities.

In view of the various biological and pharmacological activities associated with chalcones, we report the synthesis, characterization and anti-inflammatory activities of some new chalcone derivatives (9A-K), prepared by furfural and apocynin.

\section{EXPERIMENTAL}

The solvents were purified according to standard procedures prior to use and all commercial chemicals were used as received. For thin-layer chromatography (TLC) analysis,
Merck pre-coated plates (silica gel 60 F254) were used and spots were visualized under UV light. Merck silica gel 60 (230400 mesh) was used for flash column chromatography and the eluting solvents are indicated in the procedures. Melting point determinations were performed by using Mel-temp apparatus and are uncorrected. ${ }^{1} \mathrm{H}$ NMR spectra were recorded in Varian MR-400 MHz instrument. The mass spectra were recorded on Agilent ion trap MS and infrared spectra were recorded on a Perkin Elmer FT-IR spectrometer.

5-Nitrofurfural diacetate (2): A premixed solution of concentrated nitric acid $(8.6 \mathrm{~mL}, 12.2 \mathrm{~g}, 193.62 \mathrm{mmol})$ and concentrated sulphuric acid $(0.06 \mathrm{~mL}, 1.1 \mathrm{~g}, 11.2 \mathrm{mmol})$ was added drop-wise into acetic anhydride $(90 \mathrm{~mL})$ at $0{ }^{\circ} \mathrm{C}$ with stirring. To the above reaction mixture, furfural (1) (freshly distilled, $10.4 \mathrm{~mL}, 12.06 \mathrm{~g}, 125.5 \mathrm{mmol}$ ) was added dropwise over a period of $45 \mathrm{~min}$ and stirred for $1 \mathrm{~h}$ at $0{ }^{\circ} \mathrm{C}$. Water $(100 \mathrm{~mL})$ was added to the mixture and stirred at room temperature for $30 \mathrm{~min}$ to obtain a white precipitate. To the reaction contents, $10 \% \mathrm{NaOH}$ solution was added until the $\mathrm{pH}$ of the mixture reached about 2.5 and the mixture was heated at $50{ }^{\circ} \mathrm{C}$ for $1 \mathrm{~h}$. After cooling to room temperature, the white precipitate formed was filtered, washed with water, recrystallized from anhydrous ethanol and dried to obtain diacetate compound 2. Yield: $24.84 \mathrm{~g}, 82 \%$.

5-Nitrofurfural (3): A mixture of 5-nitrofurfural diacetate (2) $(10 \mathrm{~g}, 41.12 \mathrm{mmol})$ and $50 \%$ aqueous sulphuric acid (100 $\mathrm{mL}$ ) was heated to $100{ }^{\circ} \mathrm{C}$ for $10 \mathrm{~min}$. After completion of the 
reaction, checked by TLC, the reaction mixture was cooled to room temperature and extracted with ethyl acetate $(2 \times 100 \mathrm{~mL})$ and the organic layer was washed with water, brine solution and dried over anhydrous sodium sulphate, filtered and concentrated under reduced pressure to obtain 5-nitrofurfural 3. Yield: 5.10 g, $88 \%$. m.p.: $35-36{ }^{\circ} \mathrm{C}$.

(5-Nitrofuran-2-yl)methanol (4): To a stirred solution of 5-nitrofurfural (3) $(5 \mathrm{~g}, 35.44 \mathrm{mmol})$ in methanol $(100 \mathrm{~mL})$, cooled to $0{ }^{\circ} \mathrm{C}$, was added sodium borohydride $(1.47 \mathrm{~g}, 38.98$ $\mathrm{mmol}$ ) portion-wise and continued to stirred for additional 30 min. After completion of the reaction, checked by TLC, the solvent was concentrated under reduced pressure and the residue was quenched with water $(2 \mathrm{~mL})$ and extracted with cyclopentyl methylether $(4 \times 25 \mathrm{~mL})$. The organic layer was washed with water $(2 \times 30 \mathrm{~mL})$, dried over $\mathrm{Na}_{2} \mathrm{SO}_{4}$ and evaporated to obtain (5-nitrofuran-2-yl)methanol 4. Pale yellow oil; Yield: $2.33 \mathrm{~g}, 46 \% ;{ }^{1} \mathrm{H} \mathrm{NMR}\left(400 \mathrm{MHz}, \mathrm{CDCl}_{3}\right) \delta 2.50$ (br s, $1 \mathrm{H}, \mathrm{OH}), 4.73$ (s, 2H, - $\left.\mathrm{CH}_{2}\right), 6.57$ (d, $1 \mathrm{H}, J=4 \mathrm{~Hz}, \mathrm{H}_{3}$-furan), $7.30\left(\mathrm{~d}, 1 \mathrm{H}, J=4.0 \mathrm{~Hz}, \mathrm{H}_{4}\right.$-furan).

2-(Bromomethyl)-5-nitrofuran (5): To a stirred solution of triphenyl phosphine (39.40 g, $150.24 \mathrm{mmol}, 4.3 \mathrm{eq})$ in dichloromethane $(200 \mathrm{~mL})$ was added tribromoisocyanuric acid (14.05 g, $38.34 \mathrm{mmol}, 1.5 \mathrm{eq})$. After $30 \mathrm{~min}$, the alcohol (4) (5 g, $34.94 \mathrm{mmol}, 1 \mathrm{eq}$ ) was added and the suspension was stirred at room temperature for $4 \mathrm{~h}$. After completion of the reaction, the precipitated cyanuric acid was filtered off and the organic layer was washed with water $(4 \times 100 \mathrm{~mL})$ followed by brine solution. The organic layer was dried over $\mathrm{Na}_{2} \mathrm{SO}_{4}$, filtered and evaporated under reduced pressure. The obtained residue was treated with $n$-hexane and filtered through a short column packed with silica gel (100-200 mesh). Evaporation of $n$-hexane gave the desired product 5. Yellow oily liquid; Yield: $6.33 \mathrm{~g}, 84 \%$. IR $\left(\mathrm{KBr}, \mathrm{v}_{\max }, \mathrm{cm}^{-1}\right)$ : 1526 and $1345\left(\mathrm{NO}_{2}\right)$; ${ }^{1} \mathrm{H}$ NMR (400 MHz, $\left.\mathrm{CDCl}_{3}\right) \delta 4.49(\mathrm{~s}, 2 \mathrm{H}), 6.64$ (d, $J=4.0$ $\mathrm{Hz}, 1 \mathrm{H}, \mathrm{H}_{3}$-furan), 7.28 (d, $J=4.0 \mathrm{~Hz}, 1 \mathrm{H}, \mathrm{H}_{4}$-furan).

1-(4-((5-Nitrofuran-2-yl)methoxy)-3-methoxyphenyl)ethanone (7): To a solution of apocynin (6) (1.5 g, $9.02 \mathrm{mmol}$, $1.0 \mathrm{eq})$ in 2-methyl tetrahydrofuran $(25 \mathrm{~mL})$ was added potassium carbonate $(1.5 \mathrm{~g}, 10.82 \mathrm{mmol}, 1.2 \mathrm{eq})$ at room temperature and stirred for $15 \mathrm{~min}$. To the above reaction contents, compound 5 ( $1.88 \mathrm{~g}, 9.11 \mathrm{mmol}, 1.01 \mathrm{eq}$ ) was added slowly over a period of $15 \mathrm{~min}$ and heated to reflux for $1 \mathrm{~h}$. After the completion of the reaction (checked by TLC), the reaction mixture was diluted with water $(15 \mathrm{~mL})$ and stirred for $15 \mathrm{~min}$. The organic layer was washed with water $(2 \times 10 \mathrm{~mL})$ followed by brine solution, separated, dried over $\mathrm{Na}_{2} \mathrm{SO}_{4}(5 \mathrm{~g})$, filtered and evaporated under reduced pressure to obtain crude compound. The crude compound was titrated with $n$-hexane to obtain pure compound 7. Pale yellow solid; Yield: 3.65 g, $94 \%$; m.p.: $72-73{ }^{\circ} \mathrm{C} ; \mathrm{IR}\left(\mathrm{KBr}, \mathrm{v}_{\max }, \mathrm{cm}^{-1}\right)$ : 3072 (-C-H stretch, aromatic), 1681 (-C=O stretch), 1587 (-C=C-stretch), 1512 and $1338\left(-\mathrm{NO}_{2}\right.$ stretch), 1084 (-C-O stretch); ${ }^{1} \mathrm{H}$ NMR (400 MHz, DMSO$\left.d_{6}\right): \delta 2.58(\mathrm{~s}, 3 \mathrm{H}), 3.98(\mathrm{~s}, 3 \mathrm{H}), 5.20(\mathrm{~s}, 2 \mathrm{H}), 6.56(\mathrm{~s}, 1 \mathrm{H})$, $6.80(\mathrm{~s}, 1 \mathrm{H}), 7.0$ (d, $J=7.2 \mathrm{~Hz}, 1 \mathrm{H}), 7.58$ (s, $2 \mathrm{H})$; ESI-MS: $\mathrm{m} / \mathrm{z}, 292.1(\mathrm{M}+1)$.

General experimental preparation of chalcones derivatives (9A-9K): A mixture of compound 7 (100 mg, 0.343 $\mathrm{mmol})$, aromatic aldehydes $(\mathbf{8 A}-\mathbf{K})(0.346 \mathrm{mmol})$ and sodium hydroxide $(0.3 \mathrm{mmol})$ was thoroughly ground with a pestle in an open mortar $[17,18]$ at room temperature for 5-10 $\mathrm{min}$. After completion of the reaction, checked by TLC, the precipitated solid was washed with water $(4 \mathrm{~mL})$ and further recrystallized from ethanol $(2 \mathrm{~mL})$ to give the corresponding chalcone derivatives $(\mathbf{9 A}-\mathbf{K})$. Yields of the compounds varied between $86-96 \%$.

3-(4-Bromo-phenyl)-1-[3-methoxy-4-(5-nitro-furan-2ylmethoxy)phenyl]propenone (9A): Pale yellow solid; Yield: $94 \%$; m.p.: $97-98{ }^{\circ} \mathrm{C}$; IR (KBr, $\left.\mathrm{v}_{\max }, \mathrm{cm}^{-1}\right)$ : $3110(-\mathrm{C}-\mathrm{H}$ stretch, aromatic), 2942 (-C-H stretch, aliphatic), 1655 (-C=O, conjugated with $-\mathrm{C}=\mathrm{C}$ and benzene ring $), 1603,1575(-\mathrm{C}=\mathrm{C}$ of enone moiety), 1513 and 1340 (-NO $\mathrm{NO}_{2}$ stretch), 1069 (C-O stretch); ${ }^{1} \mathrm{H}$ NMR $\left(400 \mathrm{MHz}, \mathrm{CDCl}_{3}\right): \delta 3.98(\mathrm{~s}, 3 \mathrm{H}), 5.20(\mathrm{~s}$, $2 \mathrm{H}), 6.58(\mathrm{~s}, 1 \mathrm{H}), 6.80(\mathrm{~s}, 1 \mathrm{H}), 7.08(\mathrm{~d}, J=8.5 \mathrm{~Hz}, 1 \mathrm{H}), 7.58-$ $7.50(\mathrm{~m}, 5 \mathrm{H}), 7.68(\mathrm{~d}, J=5.8 \mathrm{~Hz}, 2 \mathrm{H}), 7.80(\mathrm{~d}, J=12.4 \mathrm{~Hz}$, 1H); ESI-MS: m/z, $458.1(\mathrm{M}+1)$.

3-(4-Chloro-phenyl)-1-[3-methoxy-4-(5-nitro-furan-2ylmethoxy)phenyl]propenone (9B): Yellow solid; Yield: $89 \%$; m.p.: 107-108 ${ }^{\circ} \mathrm{C}$; IR (KBr, $\left.v_{\max }, \mathrm{cm}^{-1}\right)$ : 3112 (-C-H stretch, aromatic), 2938 (-C-H stretch, aliphatic), 1654 (-C=O, conjugated with $-\mathrm{C}=\mathrm{C}$ and benzene ring), 1602, 1567 (-C=C of enone moiety), 1513 and 1349 (-NO $\mathrm{N}_{2}$ stretch), 1072 (C-O stretch); ${ }^{1} \mathrm{H}$ NMR (400 MHz, DMSO- $\left.d_{6}\right): \delta 3.85$ (s, 3H), 5.29 $(\mathrm{s}, 2 \mathrm{H}), 6.87(\mathrm{~s}, 1 \mathrm{H}), 7.28(\mathrm{~d}, J=9.5 \mathrm{~Hz}, 2 \mathrm{H}), 7.46(\mathrm{~d}, J=7.6$ $\mathrm{Hz}, 2 \mathrm{H}), 7.68$ (d, $J=15.0 \mathrm{~Hz}, 1 \mathrm{H}), 7.65(\mathrm{~d}, J=18.0 \mathrm{~Hz}, 1 \mathrm{H})$, $7.83(\mathrm{~d}, J=6.0 \mathrm{~Hz}, 1 \mathrm{H}), 7.95(\mathrm{~d}, J=8.5 \mathrm{~Hz}, 1 \mathrm{H}), 8.02$ (s, 1H), 8.06 (d, $J=8.0 \mathrm{~Hz}, 1 \mathrm{H})$; ESI-MS: m/z, $414.2(\mathrm{M}+1)$.

3-(4-Fluoro-phenyl)-1-[3-methoxy-4-(5-nitro-furan-2ylmethoxy)phenyl]propenone (9C): Off white solid; Yield: $88 \%$; m.p.: $123-124^{\circ} \mathrm{C}$; IR $\left(\mathrm{KBr}, v_{\max }, \mathrm{cm}^{-1}\right)$ : 2943 (-C-H stretch, aromatic), $1655(-\mathrm{C}=\mathrm{O}$, conjugated with $-\mathrm{C}=\mathrm{C}$ and benzene ring), 1574 (-C=C of enone moiety), 1508 and $1350\left(-\mathrm{NO}_{2}\right.$ stretch), 1024 (C-O stretch); ${ }^{1} \mathrm{H}$ NMR (400 MHz, DMSO- $\left.d_{6}\right)$ : $\delta 3.96(\mathrm{~s}, 3 \mathrm{H}), 5.20(\mathrm{~s}, 2 \mathrm{H}), 6.54(\mathrm{~d}, J=2.5 \mathrm{~Hz}, 1 \mathrm{H}), 6.78(\mathrm{~s}$, 1H), $7.04(\mathrm{~d}, J=8.0 \mathrm{~Hz}, 1 \mathrm{H}), 7.10(\mathrm{~s}, 1 \mathrm{H}), 7.13(\mathrm{~d}, J=8.0$ $\mathrm{Hz}, 1 \mathrm{H}), 7.48$ (d, $J=15.5 \mathrm{~Hz}, 1 \mathrm{H}), 7.65$ (d, $J=2.0 \mathrm{~Hz}, 2 \mathrm{H})$, $7.64(\mathrm{~d}, J=3.0 \mathrm{~Hz}, 2 \mathrm{H}), 7.78(\mathrm{~d}, J=15.5 \mathrm{~Hz}, 1 \mathrm{H})$; ESI-MS: $\mathrm{m} / \mathrm{z}, 398.3(\mathrm{M}+1)$.

1-[3-Methoxy-4-(5-nitro-furan-2-ylmethoxy)phenyl]3-(4-trifluoromethoxyphenyl)propenone (9D): Pale yellow solid; Yield: $90 \%$; m.p.: $76-77^{\circ} \mathrm{C}$; IR $\left(\mathrm{KBr}, \mathrm{v}_{\max }, \mathrm{cm}^{-1}\right): 3111$ (-C-H stretch, aromatic), $1656(-\mathrm{C}=\mathrm{O}$, conjugated with $-\mathrm{C}=\mathrm{C}$ and benzene ring), 1603, 1574 ( $\mathrm{C}=\mathrm{C}$ of enone moiety), 1507 and $1351\left(-\mathrm{NO}_{2}\right.$ stretch), 1106 (C-O stretch); ${ }^{1} \mathrm{H}$ NMR (400 MHz, DMSO-d $\left.d_{6}\right): \delta 3.97(\mathrm{~s}, 3 \mathrm{H}), 5.20(\mathrm{~s}, 2 \mathrm{H}), 6.54(\mathrm{~d}, J=3.5$ $\mathrm{Hz}, 1 \mathrm{H}), 6.78(\mathrm{~d}, J=2.0 \mathrm{~Hz}, 1 \mathrm{H}), 7.04(\mathrm{~d}, J=8.5 \mathrm{~Hz}, 1 \mathrm{H})$, $7.27(\mathrm{~d}, J=7.5 \mathrm{~Hz}, 2 \mathrm{H}), 7.51(\mathrm{~d}, J=15.5 \mathrm{~Hz}, 1 \mathrm{H}), 7.67$ (d, $J$ $=16.5 \mathrm{~Hz}, 1 \mathrm{H}), 7.68(\mathrm{~d}, J=8.0 \mathrm{~Hz}, 3 \mathrm{H}), 7.78(\mathrm{~d}, J=15.5 \mathrm{~Hz}$, 1H), ESI-MS: m/z, $464.1(\mathrm{M}+1)$.

1-[3-Methoxy-4-(5-nitro-furan-2-ylmethoxy)phenyl]3-(4-nitrophenyl)propenone (9E): Yellow solid; m.p.: 68$69{ }^{\circ} \mathrm{C}$; Yield: $94 \%$; IR (KBr, $\left.v_{\max }, \mathrm{cm}^{-1}\right)$ : 3120 (-C-H stretch, aromatic), 2944 (-C-H stretch, aliphatic), 1655 (-C=O, conjugated with $-\mathrm{C}=\mathrm{C}$ and benzene ring), $1574(\mathrm{C}=\mathrm{C}$ of enone moiety), 1517 and $1341\left(\mathrm{NO}_{2}\right), 1169$ (C-O stretch); ${ }^{1} \mathrm{H}$ NMR $\left(400 \mathrm{MHz}, \mathrm{DMSO}-d_{6}\right): \delta 3.90(\mathrm{~s}, 3 \mathrm{H}), 5.30(\mathrm{~s}, 2 \mathrm{H}), 6.88(\mathrm{~s}$, $1 \mathrm{H}), 7.28(\mathrm{~s}, 1 \mathrm{H}), 7.30(\mathrm{~d}, J=15.2 \mathrm{~Hz}, 1 \mathrm{H}), 7.65(\mathrm{~s}, 1 \mathrm{H}), 7.76$ 
$(\mathrm{d}, J=15 \mathrm{~Hz}, 1 \mathrm{H}), 7.98$ (d, $J=7 \mathrm{~Hz}, 1 \mathrm{H}), 8.18$ (d, $J=15.0$ $\mathrm{Hz}, 1 \mathrm{H}), 8.20(\mathrm{~d}, J=6.8 \mathrm{~Hz}, 2 \mathrm{H}), 8.40(\mathrm{~d}, J=6.9 \mathrm{~Hz}, 2 \mathrm{H})$; ESI-MS: $\mathrm{m} / \mathrm{z}, 425.1(\mathrm{M}+1)$.

3-(4-Methanesulfonyl-phenyl)-1-[3-methoxy-4-(5nitro-furan-2-ylmethoxy)phenyl]propenone (9F): White solid; m.p.: $130-131{ }^{\circ} \mathrm{C}$; Yield: $86 \%$; IR $\left(\mathrm{KBr}, v_{\max }, \mathrm{cm}^{-1}\right)$ : 3001 (-C-H stretch, aromatic), 1652 (-C=O, conjugated with $-\mathrm{C}=\mathrm{C}$ and benzene ring), 1599, 1565 ( $\mathrm{C}=\mathrm{C}$ of enone moiety), 1331 (-NO ${ }_{2}$ stretch), 1060 (C-O stretch); ${ }^{1} \mathrm{H}$ NMR (400 MHz, DMSO- $\left.d_{6}\right): \delta 3.10(\mathrm{~s}, 3 \mathrm{H}), 3.98(\mathrm{~s}, 3 \mathrm{H}), 5.20(\mathrm{~s}, 2 \mathrm{H}), 6.56(\mathrm{~s}$, $1 \mathrm{H}), 6.78(\mathrm{~s}, 1 \mathrm{H}), 7.06(\mathrm{~d}, J=8.4 \mathrm{~Hz}, 1 \mathrm{H}), 7.66-7.54(\mathrm{~m}, 3 \mathrm{H})$, 7.86-7.82 (m, 3H), 8.00 (d, $J=10.8 \mathrm{~Hz}, 2 \mathrm{H})$; ESI-MS: 458.3 $(\mathrm{M}+1)$.

1-[3-Methoxy-4-(5-nitro-furan-2-ylmethoxy)phenyl]3-(4-methoxyphenyl)propenone (9G): Off white solid; m.p.: 125-126 ${ }^{\circ} \mathrm{C}$; Yield: $88 \%$; IR $\left(\mathrm{KBr}, v_{\max }, \mathrm{cm}^{-1}\right): 3098$ (-C-H stretch, aromatic), 2937 (-C-H stretch, aliphatic), 1653 $(\mathrm{C}=\mathrm{C}$ of enone moiety), 1595, $1571(-\mathrm{C}=\mathrm{O}$, conjugated with $-\mathrm{C}=\mathrm{C}$ and benzene ring), $1347\left(-\mathrm{NO}_{2}\right.$ stretch $), 1161(\mathrm{C}-\mathrm{O}$ stretch); ${ }^{1} \mathrm{H}$ NMR (400 MHz, DMSO- $\left.d_{6}\right): \delta 3.82(\mathrm{~s}, 3 \mathrm{H}), 3.85$ $(\mathrm{s}, 3 \mathrm{H}), 5.28(\mathrm{~s}, 2 \mathrm{H}), 6.82(\mathrm{~s}, 1 \mathrm{H}), 7.01(\mathrm{~d}, J=8.0 \mathrm{~Hz}, 2 \mathrm{H})$, $7.25(\mathrm{~d}, J=8.1 \mathrm{~Hz}, 2 \mathrm{H}), 7.62(\mathrm{~s}, 1 \mathrm{H}), 7.70(\mathrm{~d}, J=15.0 \mathrm{~Hz}, 1 \mathrm{H})$, 7.82 (s, 1H), 7.85 (d, J = 9.0 Hz, 3H); ESI-MS: $410.3(\mathrm{M}+1)$.

3-(2,4-Dimethoxy-phenyl)-1-[3-methoxy-4-(5-nitrofuran-2-ylmethoxy)phenyl]propenone (9H): Yellow solid; m.p.: 102-103 ${ }^{\circ} \mathrm{C}$; Yield: $86 \%$; IR $\left(\mathrm{KBr}, \mathrm{v}_{\max }, \mathrm{cm}^{-1}\right): 3064$ (-C-H stretch, aromatic), 2997 (-C-H stretch, aliphatic), 1651 ( $-\mathrm{C}=\mathrm{O}$, conjugated with $-\mathrm{C}=\mathrm{C}$ and benzene ring), 1596, 1567 $\left(\mathrm{C}=\mathrm{C}\right.$ of enone moiety), $1327\left(-\mathrm{NO}_{2}\right.$ stretch $), 1057$ (C-O stretch); ${ }^{1} \mathrm{H}$ NMR (400 MHz, DMSO- $\left.d_{6}\right): \delta 3.86$ (s, 3H), 3.90 $(\mathrm{s}, 3 \mathrm{H}), 3.95(\mathrm{~s}, 3 \mathrm{H}), 5.18(\mathrm{~s}, 2 \mathrm{H}), 6.48(\mathrm{~s}, 1 \mathrm{H}), 6.52(\mathrm{~d}, J=$ $3.5 \mathrm{~Hz}, 1 \mathrm{H}), 6.54(\mathrm{~s}, 1 \mathrm{H}), 6.77(\mathrm{~s}, 1 \mathrm{H}), 7.0(\mathrm{~d}, J=8.0 \mathrm{~Hz} .1 \mathrm{H})$, $7.54(\mathrm{~d}, J=15.5 \mathrm{~Hz}, 1 \mathrm{H}), 7.58(\mathrm{~d}, J=9.0 \mathrm{~Hz}, 2 \mathrm{H}), 7.62(\mathrm{~d}$, $J=8.0 \mathrm{~Hz}, 1 \mathrm{H}), 8.04$ (d, 1H, $J=15.5 \mathrm{~Hz}, 1 \mathrm{H})$; ESI-MS: $440.1(\mathrm{M}+1)$;

3-(2,5-Dimethoxy-phenyl)-1-[3-methoxy-4-(5-nitrofuran-2-ylmethoxy)phenyl]propenone (9I): Pale yellow solid; m.p.: 121-122 ${ }^{\circ} \mathrm{C}$; Yield: $88 \%$; IR ( $\left.\mathrm{KBr}, \mathrm{v}_{\max }, \mathrm{cm}^{-1}\right)$ : 3078 (-C-H stretch, aromatic), 2935 (-C-H stretch, aliphatic), 1649 ( $-\mathrm{C}=\mathrm{O}$, conjugated with $-\mathrm{C}=\mathrm{C}$ and benzene ring $), 1599$, 1575 (-C=C of enone moiety), 1514 and $1319\left(-\mathrm{NO}_{2}\right.$ stretch), 1055 (C-O stretch); ${ }^{1} \mathrm{H}$ NMR (400 MHz, $\left.\mathrm{CDCl}_{3}\right): \delta 3.82(\mathrm{~s}$, $3 \mathrm{H}), 3.88(\mathrm{~s}, 6 \mathrm{H}), 5.30(\mathrm{~s}, 2 \mathrm{H}), 6.80(\mathrm{~s}, 1 \mathrm{H}), 7.10(\mathrm{~s}, 2 \mathrm{H}), 7.30$ $(\mathrm{s}, 2 \mathrm{H}), 7.50(\mathrm{~d}, J=16 \mathrm{~Hz}, 2 \mathrm{H}), 7.75(\mathrm{~d}, J=8.5 \mathrm{~Hz}, 2 \mathrm{H}), 8.10$ (d, $J=9.0 \mathrm{~Hz}, 1 \mathrm{H})$; ESI-MS: $440.3(\mathrm{M}+1)$;

3-(2,6-Dimethoxy-phenyl)-1-[3-methoxy-4-(5-nitrofuran-2-ylmethoxy)phenyl]propenone (9J): Yellow solid; m.p.: $127-128{ }^{\circ} \mathrm{C}$; Yield: $90 \%$; IR $\left(\mathrm{KBr}, \mathrm{v}_{\max }, \mathrm{cm}^{-1}\right): 3114$ (-C-H stretch, aromatic), 2943 (-C-H stretch, aliphatic), 1649 ( $-\mathrm{C}=\mathrm{O}$, conjugated with $-\mathrm{C}=\mathrm{C}$ and benzene ring), 1595, 1573 $\left(-\mathrm{C}=\mathrm{C}\right.$ of enone moiety), 1512 and $1320\left(-\mathrm{NO}_{2}\right.$ stretch $), 1106$ (C-O stretch); ${ }^{1} \mathrm{H}$ NMR (400 MHz, DMSO- $\left.d_{6}\right)$ : $\delta 3.92$ (s, 6H), $3.95(\mathrm{~s}, 3 \mathrm{H}), 5.18(\mathrm{~s}, 2 \mathrm{H}), 6.52(\mathrm{~s}, 1 \mathrm{H}), 6.60(\mathrm{~d}, J=6.5 \mathrm{~Hz}$, 2H), $6.77(\mathrm{~s}, 1 \mathrm{H}), 7.01(\mathrm{~d}, J=6.5 \mathrm{~Hz}, 1 \mathrm{H}), 7.28(\mathrm{~d}, J=15.5$ $\mathrm{Hz}, 1 \mathrm{H}), 7.65$ (brs, 1H), 7.98 (d, $J=9.0 \mathrm{~Hz}, 1 \mathrm{H}), 8.24$ (d, $J=$ $15.5 \mathrm{~Hz}, 1 \mathrm{H})$; ESI-MS: $440.1(\mathrm{M}+1)$;

3-(3,4,5-Triimethoxy-phenyl)-1-[3-methoxy-4-(5-nitrofuran-2-ylmethoxy)phenyl]propenone (9K): Off white solid; m.p.: 99-100 ${ }^{\circ} \mathrm{C}$; Yield: $92 \%$; IR $\left(\mathrm{KBr}, \nu_{\max }, \mathrm{cm}^{-1}\right): 3009$ (-C-H stretch, aromatic), 2940 (-C-H stretch, aliphatic), 1653 ( $-\mathrm{C}=\mathrm{O}$, conjugated with $-\mathrm{C}=\mathrm{C}$ and benzene ring), 1581,1575 (-C=C of enone moiety), $1350\left(-\mathrm{NO}_{2}\right.$ stretch), 1055 (C-O stretch); ${ }^{1} \mathrm{H}$ NMR (400 MHz, DMSO- $\left.d_{6}\right): \delta 3.70(\mathrm{~s}, 3 \mathrm{H}), 3.90$ (s, 9H), $5.30(\mathrm{~s}, 2 \mathrm{H}), 6.80(\mathrm{~s}, 1 \mathrm{H}), 7.20(\mathrm{~s}, 2 \mathrm{H}), 7.28$ (d, $J=$ $8.5 \mathrm{~Hz}, 2 \mathrm{H}), 7.60$ (s, 1H), 7.70 (d, $J=15.0 \mathrm{~Hz}, 1 \mathrm{H}), 7.88$ (s, 1H), 7.99 (d, $J=15.0 \mathrm{~Hz}, 1 \mathrm{H})$; ESI-MS: $470.3(\mathrm{M}+1)$;

Anti-inflammatory activity: A standard model system, carrageenan induced inflammatory rat model [17] was followed for the experimentation on acute inflammatory conditions. Adult wistar rats weighing between 150-200 g were used for the study. Under standard laboratory conditions, rats were maintained (temperature $25 \pm 2{ }^{\circ} \mathrm{C}$ ) with normal daily cycle $(12 / 12 \mathrm{~h})$. Before the start of experiments, the rats were made to accustom to laboratory condition for 10 days. The study was accordingly permitted by the Institutional Animal Ethical Committee (IAEC) of CPCSEA (Committee for the Purpose of Control and Supervision of Experiments on Animals). The animals were starved overnight. Diclofenac sodium (standard drug) at dose of $10 \mathrm{mg} / \mathrm{kg}$ and test compounds (9A-9K, 10 $\mathrm{mg} / \mathrm{kg}$ i.p), were controlled orally using gastric canula, $30 \mathrm{~min}$ before the carrageenan injection in sub plantar region of left hind paw. Paw edema was induced by injecting $0.1 \mathrm{~mL}$ of $1 \%$ w/v carrageenan suspended in $1 \%$ CMC into sub-plantar tissues of the left hind paw of each rat. The degree of paw thickness of all the groups was measured (in millimeters) using a vernier caliper after 1, 2 and $3 \mathrm{~h}$ of carrageenan injection.

Computational analysis: The paw thickness was measured in millimeters and presented as mean \pm SEM and were determined using analysis of variance and group means were differentiated with Tukey-Kramer Post ANOVA test. The readings were considered when $\mathrm{P}<0.01$.

\section{RESULTS AND DISCUSSION}

The synthesis of chalcone derivatives $(\mathbf{9 A - K})$ is illustrated in Scheme-I. Nitration of furfural (1) in presence of conc. $\mathrm{HNO}_{3}$ and catalytic quantity of $\mathrm{H}_{2} \mathrm{SO}_{4}$ in acetic anhydride at $0{ }^{\circ} \mathrm{C}$ for $1 \mathrm{~h}$ gave the 5 -nitrofurfural diacetate (2). Hydrolysis of diacetate 2 in presence of $20 \%$ aq. $\mathrm{H}_{2} \mathrm{SO}_{4}$ at $100{ }^{\circ} \mathrm{C}$ for 10 min produced the intermediate 5-nitrofurfural (3) [18]. Reduction of 5-nitrofurfural (3) in presence of $\mathrm{NaBH}_{4}$ in methanol at $0{ }^{\circ} \mathrm{C}$ for 30 min gave the desired (5-nitrofuran-2yl)methanol (4). Treatment of alcohol 4 with triphenylphosphine and tribromoisocyanuric acid [19] in dichloromethane at room temperature for $4 \mathrm{~h}$ produced the desired bromide intermediate (5). Coupling of bromide 5 with apocynin (6) in presence of potassium carbonate in 2-methyltetrahydrofuran at reflux for $1 \mathrm{~h}$ gave the desired product 1-(4-((5-nitrofuran2-yl)methoxy)-3-methoxyphenyl)ethanone (7). Claisen-Schmidt reaction of ethanone $\mathbf{7}$ with aromatic aldehydes $\mathbf{8 A}-\mathbf{K}$ was carried out under solvent free conditions using solid $\mathrm{NaOH}$ as catalyst at room temperature [20,21] for 5-10 min to afford chalcones 9A-9K in $86-96 \%$ yield.

Structural elucidation of the synthesized chalcones derivatives 9A-K was determined by various spectroscopic techniques like ${ }^{1} \mathrm{H}$ NMR, mass and IR spectral data. As a representative example, the ${ }^{1} \mathrm{H}$ NMR of 3-(3,4,5-triimethoxy-phenyl)-1-[3- 

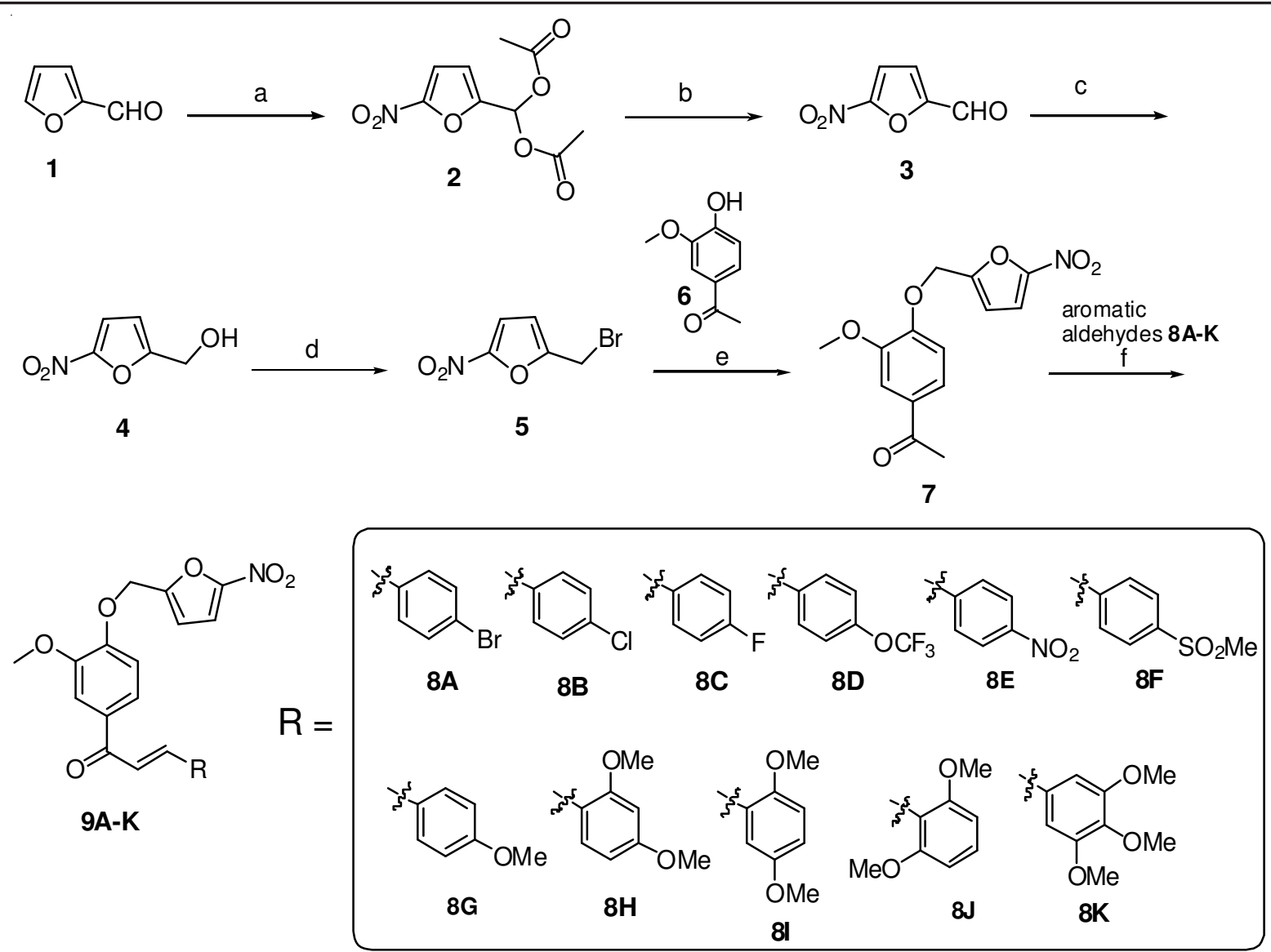

Scheme-I: Synthesis of novel chalcone derivatives $\mathbf{9 A - 9 K}$, embedded with apocynin and furan ring; Reaction conditions: a) conc; $\mathrm{HNO}_{3}$, cat.conc; $\mathrm{H}_{2} \mathrm{SO}_{4}, \mathrm{Ac}_{2} \mathrm{O}, \mathrm{O}{ }^{\circ} \mathrm{C}, 1 \mathrm{~h}, 82 \%$; b) $50 \%$ aq. $\mathrm{H}_{2} \mathrm{SO}_{4}, 100{ }^{\circ} \mathrm{C}, 10 \mathrm{~min}, 88 \%$; c) $\mathrm{NaBH}_{4}, \mathrm{MeOH}, 0{ }^{\circ} \mathrm{C}, 30 \mathrm{~min}, 46 \%$; triphenylphosphine, tribromoisocyanuric acid, room temperature, $4 \mathrm{~h}, 84 \%$; e) Apocynin, potassium carbonate, 2methyltetrahydrofuan, reflux, 1h, $94 \%$; f) aromatic aldehydes $\mathbf{8 A - K}$, sodium hydroxide, grinding, room temperature, 5-10 min, $86-96 \%$

methoxy-4-(5-nitro-furan-2-ylmethoxy)-phenyl]-propenone 9K is described here, protons resonating at $7.20 \mathrm{ppm}$ as singlet is assigned to the 3,4,5-trimethoxy phenyl ring while the protons resonating at $7.28 \mathrm{ppm}, 7.60 \mathrm{ppm}$ as doublet and singlet respectively is assigned to the vanillin ring. The singlet signals with one proton integration resonating at 6.80 and 7.88 ppm corresponds to the furan ring. The characteristic olefin protons resonating at $7.70 \mathrm{ppm}$ and $7.99 \mathrm{ppm}$ as doublets with $J=$ $15.5 \mathrm{~Hz}$ indicates the ' $E$ ' isomeric form of chalcone. Similarly, the ${ }^{1} \mathrm{H}$ NMR spectra of the remaining chalcone derivatives are in agreement with the desired structures.

The mass spectra of the compounds showed $(\mathrm{M}+1)$ peaks and are in agreement with their molecular formulae. The IR spectra of the compounds $\mathbf{9 A}-\mathbf{K}$ represented the characteristic peaks that comply with the desired functional group in the structure. The characteristic $\alpha, \beta$-unsaturated carbonyl stretching bands appeared in the regions $1606-1570 \mathrm{~cm}^{-1}(-\mathrm{C}=\mathrm{C}$ of enone moiety) and 1664-1648 $\mathrm{cm}^{-1}(-\mathrm{C}=\mathrm{O}$, conjugated with $-\mathrm{C}=\mathrm{C}$ and benzene ring).

Anti-inflammatory activity: The results of the anti-inflammatory activity (dosage: $10 \mathrm{mg} / \mathrm{Kg}$ po) of the synthesized chalcone derivatives $\mathbf{9 A - K}$ is presented in Table-1. Compounds 9D, 9C, 9F, 9K and 9E bearing $\mathrm{R}=$-4-trifluoromethoxyphenyl, -4-fluoro-phenyl, 4-sulphonyl methyl phenyl, 3,4,5trimethoxy phenyl and 4-nitro-phenyl exhibited significant

\begin{tabular}{|c|c|c|c|}
\hline \multicolumn{4}{|c|}{$\begin{array}{l}\text { TABLE-1 } \\
\text { RESULTS OF ANTI-INFLAMMATORY ACTIVITY OF } \\
\text { HYDRAZIDE-HYDRAZONE DERIVATIVES (9A-9K) }\end{array}$} \\
\hline Treatments & $1 \mathrm{~h}$ & $2 \mathrm{~h}$ & $3 \mathrm{~h}$ \\
\hline Carrageenan control & $0.98 \pm 0.09$ & $1.25 \pm 0.12$ & $2.55 \pm 0.12$ \\
\hline 9A & - & - & - \\
\hline 9B & - & - & - \\
\hline 9C & $0.68 \pm 0.48$ & $0.88 \pm 0.44$ & $0.98 \pm 0.28$ \\
\hline 9D & $0.70 \pm 0.64$ & $0.90 \pm 0.43$ & $1.08 \pm 0.22$ \\
\hline 9E & $0.56 \pm 0.18$ & $0.72 \pm 1.58$ & $0.78 \pm 0.86$ \\
\hline $9 F$ & $0.62 \pm 0.50$ & $0.82 \pm 0.34$ & $0.88 \pm 0.22$ \\
\hline 9G & $0.44 \pm 0.80$ & $0.56 \pm 1.18$ & $0.62 \pm 0.24$ \\
\hline $9 H$ & $0.38 \pm 1.12$ & $0.62 \pm 0.88$ & $0.52 \pm 0.21$ \\
\hline 9I & $0.40 \pm 0.94$ & $0.50 \pm 1.15$ & $0.48 \pm 0.40$ \\
\hline 9J & $0.38 \pm 0.78$ & $0.48 \pm 0.64$ & $0.60 \pm 0.38$ \\
\hline $9 K$ & $0.60 \pm 0.75$ & $0.75 \pm 0.98$ & $0.85 \pm 0.82$ \\
\hline $\begin{array}{l}\text { Diclofenac sodium } \\
\quad(10 \mathrm{mg} / \mathrm{kg})\end{array}$ & $0.75 \pm 0.12$ & $0.95 \pm 0.12$ & $1.16 \pm 0.11$ \\
\hline
\end{tabular}

anti-inflammatory activity while the compounds 9G, 9H, 9I and 9J bearing 4-methoxy-phenyl, 2,4-dimethoxy phenyl, 2,5dimethoxy phenyl and 2,6-dimethoxy phenyl displayed moderate anti-inflammatory activity. Furthermore, chalcones, 9A and 9B bearing 4-bromo-phenyl and 4-chloro-phenyl group was found to be inactive. 


\section{Conclusion}

In conclusion, we have described the synthesis and characterization of chalcone derivatives 1-[3-methoxy-4-(5-nitrofuran-2-ylmethoxy)-phenyl]-3-(substituted phenyl)-propenone derivatives 9A-9K utilizing commercially available furfural and apocynin. The results of the anti-inflammatory activity of these compounds revealed that, compounds 9D, 9C, 9F, 9K and $9 \mathrm{E}$ bearing $\mathrm{R}=$-4-trifluoromethoxy-phenyl, -4-fluorophenyl, 4-sulphonyl methyl phenyl, 3,4,5-trimethoxy phenyl and 4-nitro-phenyl exhibited significant anti-inflammatory activity.

\section{REFERENCES}

1. N.K. Sahu, S.S. Balbhadra, J. Choudhary and D.V. Kohli, Curr. Med. Chem., 19, 209 (2012); https://doi.org/10.2174/092986712803414132.

2. A. Kamal, S. Prabhakar, M. Janaki Ramaiah, P. Venkat Reddy, C. Ratna Reddy, A. Mallareddy, N. Shankaraiah, T. Lakshmi Narayan Reddy, S.N.C.V.L. Pushpavalli and M. Pal-Bhadra, Eur. J. Med. Chem., 46, 3820 (2011); https://doi.org/10.1016/i.ejmech.2011.05.050.

3. N. Ahmed, N.K. Konduru, S. Ahmad and M. Owais, Eur. J. Med. Chem., 82, 552 (2014);

https://doi.org/10.1016/j.ejmech.2014.06.009.

4. G.D. da Silva, M.G. da Silva, E.M.P.V.E. Souza, A. Barison, S.C. Simões, F.P. Varotti, L.A. Barbosa, G.H.R. Viana and J.A.F.P. Villar, Molecules, 17, 10331 (2012);

https://doi.org/10.3390/molecules 170910331.

5. B. Insuasty, J. Ramírez, D. Becerra, C. Echeverry, J. Quiroga, R. Abonia, S.M. Robledo, I.D. Velez, Y. Upegui, J.A. Munoz, V. Ospina, M. Nogueras and J. Cobo, Eur. J. Med. Chem., 93, 401 (2015); https://doi.org/10.1016/j.ejmech.2015.02.040.

6. C. Reichwald, O. Shimony, U. Dunkel, N. Sacerdoti-Sierra, C.L. Jaffe and C. Kunick, J. Med. Chem., 51, 659 (2008); https://doi.org/10.1021/jm7012166.

7. N. Yadav, S.K. Dixit, A. Bhattacharya, L.C. Mishra, M. Sharma, S.K. Awasthi and V.K. Bhasin, Chem. Biol. Drug Des., 80, 340 (2012); https://doi.org/10.1111/j.1747-0285.2012.01383.x.

8. L.B. Salum, W.F. Altei, L.D. Chiaradia, M.N.S. Cordeiro, R.R. Canevarolo, C.P.S. Melo, E. Winter, B. Mattei, H.N. Daghestani, M.C. Santos-Silva, T.B. Creczynski-Pasa, R.A. Yunes, J.A. Yunes, A.D. Andricopulo, B.W. Day, R.J. Nunes and A. Vogt, J. Med. Chem., 63, 501 (2013); https://doi.org/10.1016/j.ejmech.2013.02.037.
9. Y.L.N. Murthy, K.P. Suhasini, A.S. Pathania, S. Bhushan and Y.N. Sastry, Eur. J. Med. Chem., 62, 545 (2013); https://doi.org/10.1016/j.ejmech.2013.01.027.

10. H. Sharma, S. Patil, T.W. Sanchez, N. Neamati, R.F. Schinazi and J.K. Buolamwini, Bioorg. Med. Chem., 19, 2030 (2011); https://doi.org/10.1016/i.bmc.2011.01.047.

11. P.M. Sivakumar, P.K. Prabhakar and M. Doble, Med. Chem. Res., 20, 482 (2011); https://doi.org/10.1007/s00044-010-9342-1.

12. Z. Liu, L. Tang, P. Zou, Y. Zhang, Z. Wang, Q. Fang, L. Jiang, G. Chen, Z. Xu, H. Zhang and G. Liang, Eur. J. Med. Chem., 74, 671 (2014); https://doi.org/10.1016/j.ejmech.2013.10.061.

13. S.A. Carvalho, L.O. Feitosa, M. Soares, T.E.M.M. Costa, M.G. Henriques, K. Salomão, S.L. de Castro, M. Kaiser, R. Brun, J.L. Wardell, S.M.S.V. Wardell, G.H.G. Trossini, A.D. Andricopulo, E.F. da Silva and C.A.M. Fraga, Eur. J. Med. Chem., 54, 512 (2012); https://doi.org/10.1016/j.ejmech.2012.05.041.

14. K.V. Sashidhara, S.R. Avula, V. Mishra, G.R. Palnati, L.R. Singh, N. Singh, Y.S. Chhonker, P. Swami, R.S. Bhatta and G. Palit, Eur. J. Med. Chem., 89, 638 (2015); https://doi.org/10.1016/i.ejmech.2014.10.068

15. P.S. Bhale, S.B. Dongare and U.B. Chanshetti, Res. J. Chem. Sci., 3, 38 (2013).

16. B.A. Konan, K.G.M. Bouafou, K.L. Kouakou, N.M. Bléyéré, K.A. Amonkan, V.J. Zannou-Tchoko, M.-L. Berthe Ahui and Y.J. Datté, Int. J. Life Sci. Pharm. Res., 2, 82 (2012); https://doi.org/10.5963/LSMR0204005

17. A.L.V. Kumar Reddy and N.E. Kathale, Orient. J. Chem., 33, 971 (2017); https://doi.org/10.13005/ojc/330250.

18. H. Jin, Y. Geng, Z. Yu, K. Tao and T. Hou, Pestic. Biochem. Physiol., 93, 133 (2009); https://doi.org/10.1016/j.pestbp.2009.01.002.

19. S.C.V. de Andrade and C.S.M. de Mattos, J. Braz. Chem. Soc., 25, 975 (2014).

20. N.M. Rateb and H.F. Zohdi, Synth. Commun., 39, 2789 (2009); https://doi.org/10.1080/00397910802664244.

21. N.S. Rao, C. Kistareddy, B. Balram and B. Ram, Der Pharma Chem., 4, 2408 (2012) 